\title{
Fourier spectroscopy of ultrashort laser pulses
}

\author{
Scott D. Bergeson \\ scott.bergeson@byu.edu \\ Justin Peatross \\ peat@byu.edu
}

Follow this and additional works at: https://scholarsarchive.byu.edu/facpub

Part of the Astrophysics and Astronomy Commons, and the Physics Commons

\section{Original Publication Citation}

J. Peatross and S. D. Bergeson, "Fourier spectroscopy of ultrashort laser pulses," Am. J. Physics 74,842 - $845(26)$

\section{BYU ScholarsArchive Citation}

Bergeson, Scott D. and Peatross, Justin, "Fourier spectroscopy of ultrashort laser pulses" (2006). Faculty Publications. 297.

https://scholarsarchive.byu.edu/facpub/297

This Peer-Reviewed Article is brought to you for free and open access by BYU ScholarsArchive. It has been accepted for inclusion in Faculty Publications by an authorized administrator of BYU ScholarsArchive. For more information, please contact ellen_amatangelo@byu.edu. 


\title{
APPARATUS AND DEMONSTRATION NOTES
}

\author{
Jeffrey S. Dunham, Editor \\ Department of Physics, Middlebury College, Middlebury, Vermont 05753
}

\begin{abstract}
This department welcomes brief communications reporting new demonstrations, laboratory equipment, techniques, or materials of interest to teachers of physics. Notes on new applications of older apparatus, measurements supplementing data supplied by manufacturers, information which, while not new, is not generally known, procurement information, and news about apparatus under development may be suitable for publication in this section. Neither the American Journal of Physics nor the Editors assume responsibility for the correctness of the information presented. Submit materials to Jeffrey $\mathrm{S}$. Dunham, Editor.
\end{abstract}

\section{Fourier spectroscopy of ultrashort laser pulses}

\author{
J. Peatross ${ }^{\mathrm{a})}$ and S. D. Bergeson \\ Department of Physics and Astronomy, Brigham Young University, Provo, Utah 84602
}

(Received 4 April 2006; accepted 2 June 2006)

\begin{abstract}
We describe a Fourier-transform spectrometer appropriate for use in an undergraduate optics laboratory. The modular design, which uses off-the-shelf equipment, is suitable for determining the spectra of ultrashort pulsed and continuous light sources. The spectrometer does not require equal step sizes for the motion of the mirror. An algorithm interpolates the phase of both a reference and an unknown light beam at equal intervals before performing the Fourier transform. The interferometer scan and the phase and spectral analysis are performed in a few seconds, making the apparatus a useful tool for teaching concepts of temporal coherence and Fourier spectroscopy. (C) 2006 American Association of Physics Teachers.
\end{abstract}

[DOI: 10.1119/1.2218358]

\section{INTRODUCTION}

Fourier-transform spectroscopy is an important tool in many fields of research and has the advantage of high light throughput and sensitivity because all wavelengths are measured simultaneously. It also makes a good teaching tool for introducing students to the concepts of temporal coherence and its connection to the optical spectrum. ${ }^{1-4}$ A Fouriertransform spectrometer (FTS) measures the output intensity from a Michelson interferometer as the position of one of the retroreflecting mirrors is moved. The intensity must be sampled with an accurately known phase interval (determined by the mirror position) in order to transform the signal and obtain the input frequency spectrum. In addition, the signal should be sampled with uniform phase intervals so that a fast Fourier transform (FFT) can be employed.

Ensuring that measurements take place at equal-phase intervals can be difficult. ${ }^{4,5}$ One way to perform equal-phase measurements is to move the interferometer mirror at a constant velocity and to sample the output at equal time intervals. Another way employs a narrowband $\mathrm{He}-\mathrm{Ne}$ laser that copropagates with the input signal, together with a sophisticated control system that records the measured intensity at preprogrammed fractions of the $\mathrm{He}-\mathrm{Ne}$ interference fringe pattern.

Several years ago Jim Brault suggested that it is not necessary to control the motion of the interferometer mirror as long as it moves monotonically in one direction. ${ }^{6}$ Rather than use the reference beam to control (or verify) the translation of the mirror, it can be used to recover the absolute phase of a distorted measurement that is sampled at unequal intervals. High-resolution spectrometers at many research laboratories now use Brault's technique.

In this paper we describe a simple implementation of this technique constructed with inexpensive off-the-shelf components and based on a LABVIEW data acquisition system. We simultaneously sample the interference pattern of an unknown light source together with that of a reference laser. We utilized a simple interpolation routine to resample the interferograms at equal-phase intervals before performing the Fourier transform.

We have used the interferometer to measure the spectrum of femtosecond laser pulses. The result is in excellent agreement with an independent spectral measurement using a standard grating spectrometer. Although femtosecond laser systems are relatively expensive, they are increasingly common in university physics laboratories. Ultrashort pulses are an interesting light source for introducing students to the concepts of temporal coherence and to Fourier spectroscopy. In particular, it is easy for students to appreciate why the fringe amplitude decreases as the mirror is displaced. We can visualize two identical, short wave packets $(0.01 \mathrm{~mm}$ long) created at the initial encounter with the beam splitter. The two pulses move down the arms of the interferometer and back for a second encounter where interference is possible. If the path lengths in the arms are sufficiently different to cause the two pulses to arrive one after the other, we expect no interference. This behavior gives an intuitive feel as to why the coherence function diminishes when the path lengths in the interferometer differ. 


\section{FOURIER-TRANSFORM SPECTROSCOPY}

The signal $s$ measured at the output of the interferometer varies with the roundtrip time delay $\tau$ as ${ }^{8}$

$$
\mathrm{s}(\tau) \propto 1+\operatorname{Re} \gamma(\tau),
$$

where

$$
\gamma(\tau) \equiv \frac{\int_{-\infty}^{+\infty} I(\omega) e^{-i \omega \tau} d \omega}{\int_{-\infty}^{+\infty} I(\omega) d \omega} .
$$

$I(\omega)$ is the spectral intensity of the light source in terms of the optical angular frequency $\omega$. The magnitude of the coherence function $|\gamma(\tau)|$ takes on values from zero to one and is equal to the fringe visibility. It goes to zero as the time delay $\tau$ between the paths increases. The amount of time delay necessary to reduce the fringe visibility substantially is called the coherence time. For a well-compressed ultrashort pulse, the magnitude of the coherence time is comparable to the duration of the pulse.

When teaching these concepts to students, we have often encountered resistance to the notion that the measured output from the interferometer is independent of whether an ultrashort pulse is chirped, that is, if the different frequency components are spread out in time giving rise to a longer pulse duration. If the duration of the pulse is lengthened significantly, say by acquiring chirp in a thick piece of glass before entering the interferometer, the fringe visibility diminishes with mirror displacement in exactly the same manner. Even though chirped pulses may overlap significantly in time when they re-encounter each other at the beam splitter, the oscillations in the measured signal as a function of path delay are no different than for compressed pulses. This wellknown result is the reason that the temporal profile of short laser pulses is characterized using nonlinear techniques such as autocorrelation or frequency-resolved optical gating. ${ }^{9}$ In Sec. V, we demonstrate experimentally that chirping of the ultrashort pulses does not influence the result.

\section{INTERFEROMETER SETUP}

We constructed a basic Michelson interferometer using an uncoated optical flat as the beam splitter (Newport, part \# 10QB20). The interferometer fits on a $1^{\prime} \times 1^{\prime}$ optical breadboard (see Fig. 1). Reflections occur at both surfaces of the beam splitter, resulting in dual beams emerging from the interferometer (after the light from each arm re-encounters the beam splitter). The beams are well separated due to the thickness of the glass, so that one can be retained and the other discarded using an iris. The light in one arm of the interferometer makes three trips through the optical flat before heading to the detector. A second identical optical flat is placed in the other arm of the interferometer so that both beams make three trips through glass. Each arm of the interferometer employs a silver-coated flat mirror as a retroreflector. One mirror is placed on a translation stage (Melles-Griot, part \# 07TNS037), which we set to move at $\approx 1 \mathrm{~mm} / \mathrm{s} \mathrm{dur-}$ ing scanning. The output of the interferometer is sent to two detectors: one that detects the light under study and one that detects a reference beam. We used a narrow collimated $\mathrm{He}-\mathrm{Ne}$ laser as the reference beam by sending it through the

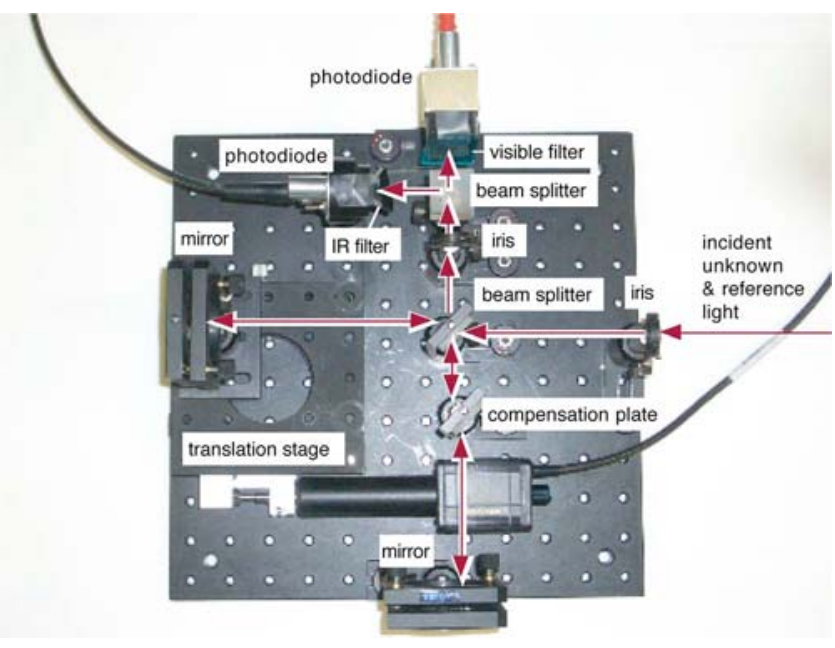

Fig. 1. Michelson interferometer outfitted with a translation stage on one arm (horizontal in photo). The output is split and sent to two photodiodes, one for measuring the reference signal and the other for measuring the signal of an unknown beam.

device several millimeters above the beam of the light under study. In this case, a pickoff mirror separates the beams at the output for individual detection.

For our study of femtosecond laser pulses, we used the residual green pump light from the femtosecond oscillator as the reference beam, which was very convenient for alignment of the interferometer. Our ultrashort pulses are generated in a self-mode-locked Ti:sapphire cavity (KMLabs) pumped by $4.5 \mathrm{~W}$ of $532 \mathrm{~nm}$ laser light (Coherent Verdi). A few milliwatts of green pump light emerges collinear with the ultrashort pulses. The 800-nm ultrashort pulses are not visible to the eye. It is convenient to rely on the collinear green pump light for interferometer alignment and to confirm the presence of wide fringes. At the output of the interferometer we installed a beam-splitter cube to direct the signal into two separate photodiodes (Thorlabs BS012). An IR-blocking filter (Schott BG-39) was placed in front of one detector, and a visible-blocking filter (Schott RG-715) was placed in front of the other detector. Thus, one detector saw $532 \mathrm{~nm}$ reference light, and the other saw the train of ultrashort pulses. The repetition rate of the short pulses is approximately $100 \mathrm{MHz}$, which is very rapid compared to the digitizing rate used during interferometer scans.

Figure 2 shows the signal measured by the two detectors as the length of one arm of the interferometer is scanned. A LABVIEW program starts the motor that scans the moving mirror a few millimeters at a rate of $1 \mathrm{~mm} / \mathrm{s}$. Through a process of trial and error, the initial motor position is chosen

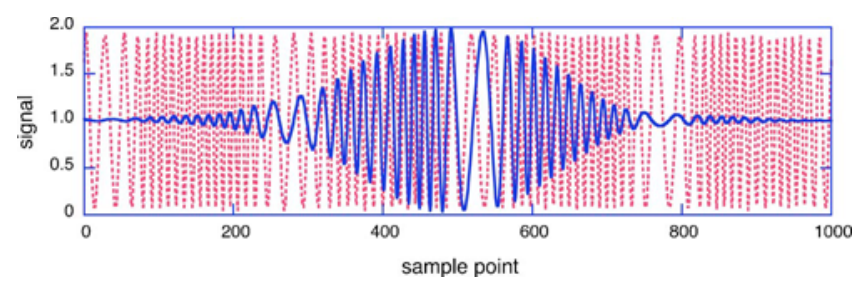

Fig. 2. Measured signal for an ultrashort IR pulse train (solid line) and for a coherent 532-nm source (dotted line) as the mirror in the interferometer is scanned. Variations in the scan velocity cause the fringes to be nonuniform. 
manually so that the peak of the interference pattern from the ultrashort pulse laser is approximately centered in the scan. While the mirror is scanning, the LABVIEW program measures the two photodiode signals using a digitizing card (National-Instruments AT-MIO-16E-10). The photodiodes are each terminated with a $1-\mathrm{k} \Omega$ resistor and their voltages sampled at $50 \mathrm{kHz}$.

We did not keep track of motor position during scanning, but rather plotted the voltages from the signal and reference light sources as a function of sample points. The curves in Fig. 2 each represent 1000 samples. The fringes for both the 532-nm reference beam and the beam of ultrashort pulses are seen. Each photodiode signal has been normalized by its own average signal such that it oscillates around a value of unity. The finite coherence length of the ultrashort pulses is readily apparent in Fig. 2. Also apparent is the artificial stretching and compressing of the fringes due to unevenness of the translation during scanning. The program allows the student to zoom in on the fringe pattern, thus truncating the data record, speeding up the FFT, but also reducing the spectral resolution. This tradeoff is particularly noticeable in the Fourier transform of the 532-nm reference beam.

\section{REMOVAL OF SCANNING ERRORS}

Although our measurement is compromised by imperfections in the translation stage movement, the mirror translation is monotonic as long as it moves with sufficient velocity $(1 \mathrm{~mm} / \mathrm{s})$ in one direction. The uneven sampling of the fringe phases must be corrected before the spectrum of the light can be recovered. As mentioned, we do not address the mechanical source of the error as others have done, either by using sophisticated actuator air bearings, electronics that record measurements at fractional intensities of the reference laser fringes, or constant-velocity actuator feedback control. Instead, we unravel the phase errors using a method similar to the one reported in Ref. 6. The method is straightforward and transparent to the student.

We first subtract one from the normalized reference signal so that it oscillates about zero. Rather than try to extract phase information at all points on the curve, we infer the phases of the sampling points using only the zero crossings of the reference signal. We find the zero-crossing points through linear interpolation. The zero crossings generally fall between data samples. Linear interpolation is adequate for estimating zero-crossing locations, because a sinusoidal function is linear in its argument near zero. The analysis produces a two-column array: one column with the zerocrossing phase (incremented by $\pi$ ) and the other with a fractional number of sample points to where each zero is estimated to occur. We use a cubic-spline fit to the zero-crossing points and assign a phase for all data points, including the majority of points that fall in between zero crossings. Finally, we do a linear interpolation to resample both the reference and the unknown wave forms at equal-phase intervals, which produces data suitable for Fourier analysis. Many standard software packages have precompiled FFT commands. Alternatively, we could avoid the resampling step and simply supply the original data points with their assigned phases to a Fourier-transform algorithm that accepts nonuniform sampling.

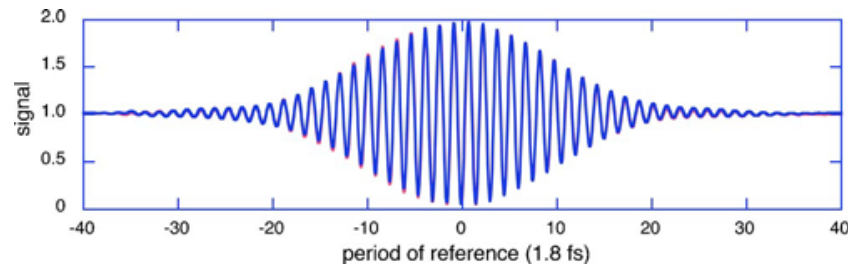

Fig. 3. Phase-compensated measurement for an ultrashort IR pulse train. Superimposed is a measurement of the same pulse train after undergoing strong dispersion, that is, chirping. The two curves are nearly indistinguishable and appear in the plot as a single curve.

\section{SPECTRAL MEASUREMENT OF AN ULTRASHORT PULSE}

Figure 3 shows the measured interference pattern for the ultrashort laser pulses after the scanning errors were removed. The fringes are plotted as a function of the time delay as measured in units of the reference-beam period rather than sample point as in Fig. 2. Visually, the coherence time is on the order of 10 periods of the reference laser (1.8 fs for $532 \mathrm{~nm}$ light), which indicates that the coherence time is about $18 \mathrm{fs}$. As is well known, ${ }^{8}$ the spectral intensity can be extracted from Eqs. (1) and (2) by taking the Fourier transform of $s(\tau)$. Figure 4 shows the result of the Fourier transform for the curve in Fig. 3. An independent measurement of the spectrum obtained with a separate spectrometer (Ocean Optics USB2000) is shown for comparison, with good agreement.

As mentioned in Sec. II, the measured fringes and the resulting spectra are independent of any chirp that might be introduced into the pulses before entering the interferometer. Indeed, the beam splitter and compensation plate themselves introduce a significant amount of chirp within the instrument. This chirp has no effect on the result as long as the amount of chirping in the two arms is the same. We demonstrated this principle by placing a 2-cm-thick piece of glass in the pulsed laser beam before it arrives at the interferometer. Fig-

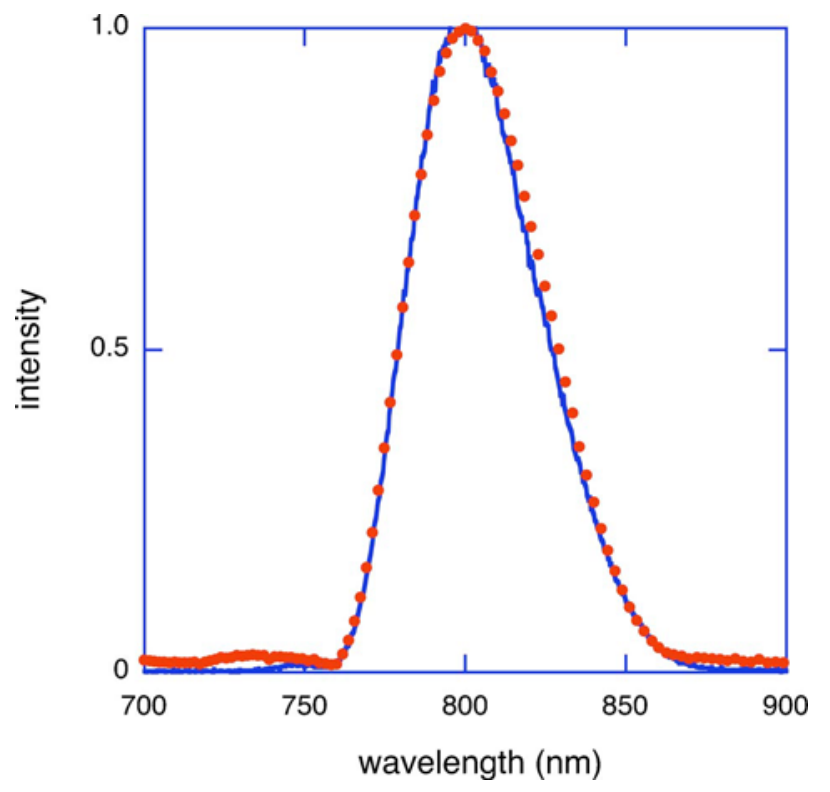

Fig. 4. The measured spectrum recovered through Fourier spectroscopy (dots) compared to that obtained using a grating spectrometer (solid line). 


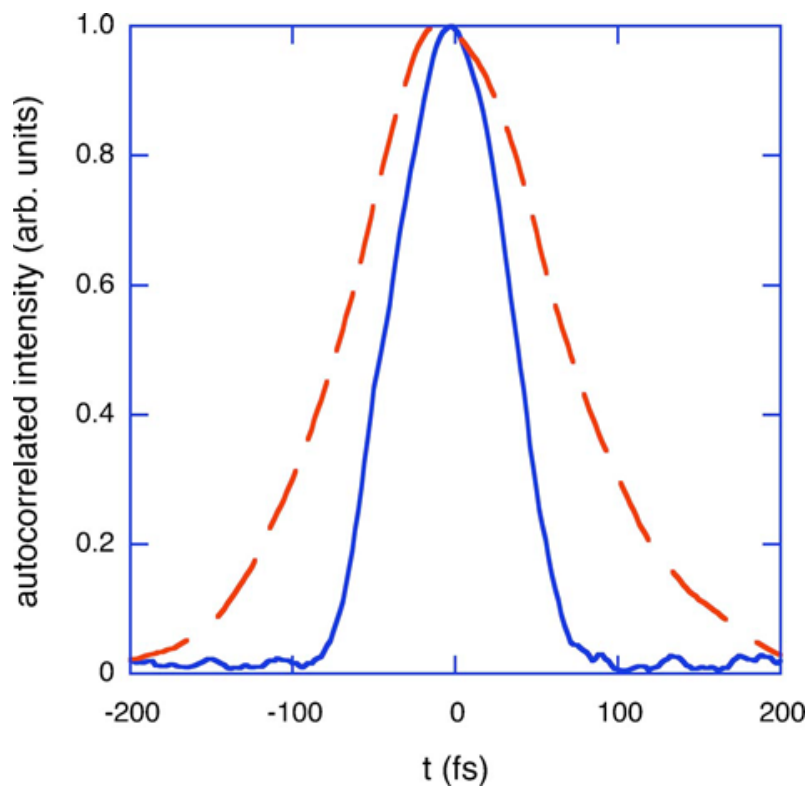

Fig. 5. Pulse-duration measurement using a nonlinear autocorrelation technique (solid line). A significantly longer pulse duration results when the ultrashort pulses travel through a thick piece of glass (long dashed line).

ure 5 shows a separate autocorrelation measurement, which characterizes the pulse duration after having been sent through a laser amplification system. The pulse duration increases by at least a factor of 2 when the glass is inserted, a conservative estimate given spectral gain narrowing in the laser amplifier. Nonetheless, the fringe pattern measured in the Michelson interferometer is unaffected. Figure 3 shows two traces of the interference fringes: one with and one without the glass inserted in the beam. The traces are virtually indistinguishable, lying precisely on top of each other to within experimental error. Of course, the resulting spectra are also identical.

\footnotetext{
${ }^{a}$ Electronic mail: peat @byu.edu

${ }^{1}$ A. R. Harvey, M. Begbie, and J. J. Padgett, "Stationary Fourier transform spectrometer for use as a teaching tool," Am. J. Phys. 62, 1033-1036 (1994).

${ }^{2}$ R. G. Layton and J. K. Brower, "Fourier-transform spectroscopy using any old Michelson interferometer," Am. J. Phys. 43, 180 (1975).

${ }^{3}$ W. C. Oelfke, "Fourier spectroscopy: A simple analysis technique," Am. J. Phys. 43, 786-789 (1975).

${ }^{4}$ D. R. Matthys and F. L. Pedrotti, "Fourier transforms and the use of a microcomputer in the advanced undergraduate laboratory," Am. J. Phys. 50, 990-995 (1982).

${ }^{5}$ J. C. Brasunas and G. M. Cushman, "A simple scanning mirror mechanism for a Fourier transform spectrometer," Rev. Sci. Instrum. 69, 16371641 (1998).

${ }^{6}$ J. W. Brault, "New approach to high-precision Fourier transform spectrometer design," Appl. Opt. 35, 2891-2896 (1996).

${ }^{7}$ C. J. Sansonetti, NIST, personal communication (2004). Spectroscopy laboratories using Brault's method include NIST, Imperial College London, NASA, and KPNO.

${ }^{8}$ G. R. Fowles, Introduction to Modern Optics, 2nd ed. (Dover, Mineola, NY, 1968), pp. 80-82.

${ }^{9}$ J.-C. Diels, Ultrashort Laser Pulse Phenomena: Fundamentals, Techniques, and Applications on a Femtosecond Time Scale (Academic, San Diego, 1996).
}

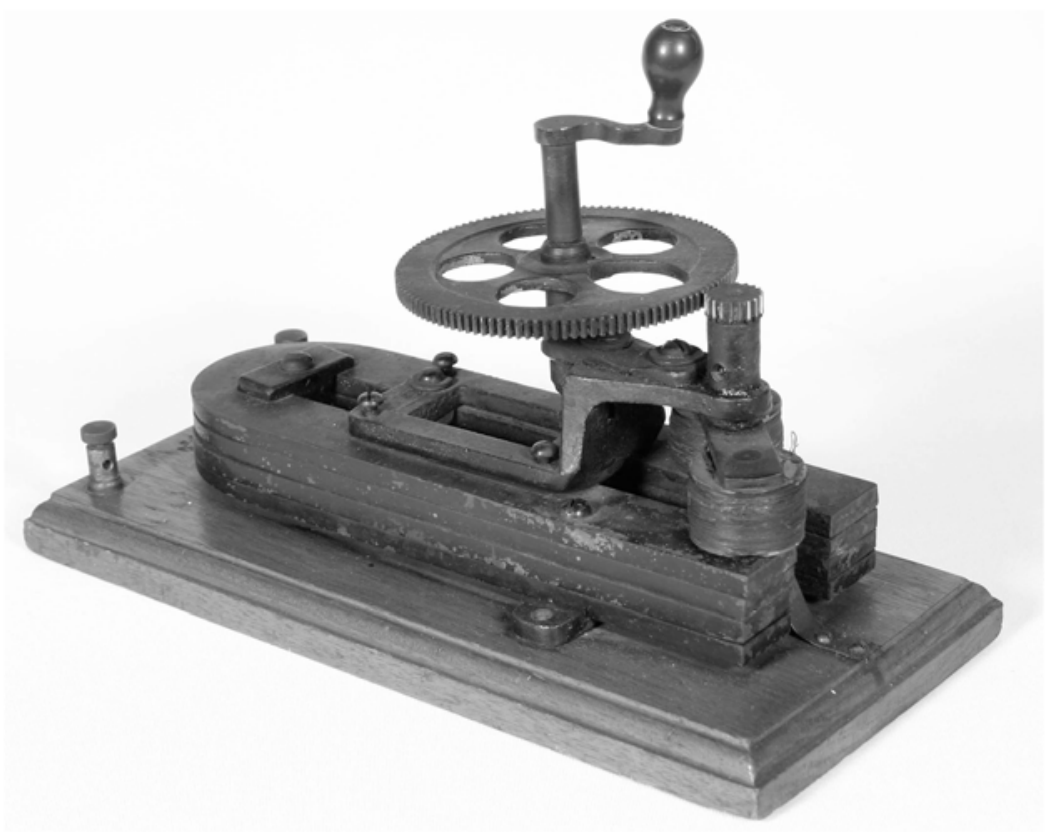

Magneto-Electric Machine. In this device, a pair of coils wound with many turns of fine wire is rotated in the magnetic field produced by a permanent magnet. Gearing allows the coils to cut the field lines rapidly, thus producing a high voltage. This design dates from the 1840s, although this example dates from the years 1882-1893. The manufacturer was Alfred P. Gage (1836-1903), who taught physics in the English High School in Boston, and wrote physics textbooks. He was involved in the early movement to include experimental work in the high school curriculum, and then went into the business of making apparatus that could be afforded by these schools. If he had lived in the 1950s he would have been a pioneer in the PSSC physics course. (Photograph and Notes by Thomas B. Greenslade, Jr., Kenyon College) 